\title{
Rescuing from toxicity
}

DOI:

10.1038/nrd2407
Parkinson's disease (PD) is characterized by the development of misfolded, $\alpha$-synuclein-containing protein aggregates and inclusions (Lewy bodies) that are responsible for cellular toxicity. Therefore modulating inclusion formation could represent an approach for therapeutic intervention. Kazantsev and colleagues, writing in Science, have shown that inhibitors of sirtuin 2 (SIRT2) - a member of the histone deacetylase family of enzymes rescues $\alpha$-synuclein-mediated toxicity in models of PD by increasing inclusion formation.

The authors previously identified a compound - known as B 2 - that increases the size of intracellular $\alpha$-synuclein inclusions from numerous small aggregates to larger, less toxic inclusions. B2 decreased toxicity in cell-based models of PD and Huntington's disease, although the target was not identified. In the present study, B2 was found to be a weak, but selective inhibitor of SIRT2. By screening a library of 200 structural analogues of B2 and other aggregation modifiers, the authors identified AGK2, a more potent inhibitor.

The authors next conducted a series of assays to determine the effect of this compound on SIRT2 activity. Treatment of purified $\alpha$-tubulin - the native substrate of SIRT2 - with AGK2 led to an increase in acetylated tubulin, demonstrating the deacetylation activity of the compound. In transfected HeLa cells, AGK2 inhibited the activity of SIRT2 that had been folded and processed by the intracellular machinery. An increase in acetylated tubulin was also observed, showing that AGK2 could enter cells and act on endogenous SIRT2 in its native environment.

AGK2 and a lower potency analogue, AK-1 reduced $\alpha$-synuclein cytotoxicity in a transfected human neuronal cell line, which correlated with the formation of enlarged inclusions. To determine whether AGK2 and $\mathrm{AK}-1$ protected dopaminergic neurons from $\alpha$-synuclein-induced toxicity, the authors examined $\alpha$-synuclein-dependent dopaminergic cell death in primary midbrain cultures. Cultures treated with B2, AK-1 or AGK2 contained more tyrosine-hydroxylase-positive (a marker of dopaminergic cells) neurons. Last, in a Drosophila melanogaster model of PD, control flies exhibited a loss of tyrosinehydroxylase-positive neurons, whereas flies fed with increasing doses of either AK-1 or AGK2 had a dramatic dose-dependent rescue of dorsomedial neurons.

Although SIRT2 inhibitors need to be tested in higher models, this study has identified that SIRT2 inhibition rescues $\alpha$-synuclein mediated cytotoxicity, possibly via inclusion enlargement - a mechanism by which a concomitant reduction in total surface area of inclusions could minimize aberrant interactions with cellular proteins. So, SIRT2 targeting might be therapeutically beneficial in PD and other conditions in which aggregation of misfolded proteins is central to disease pathogenesis.

Charlotte Harrison

ORIGINAL RESEARCH PAPER Fleming Outeiro, T. et al. Sirtuin-2 inhibitors rescue $\alpha$-synuclein-mediated toxicity in models of

Parkinson's disease. Science 317, 516-519 (2007)

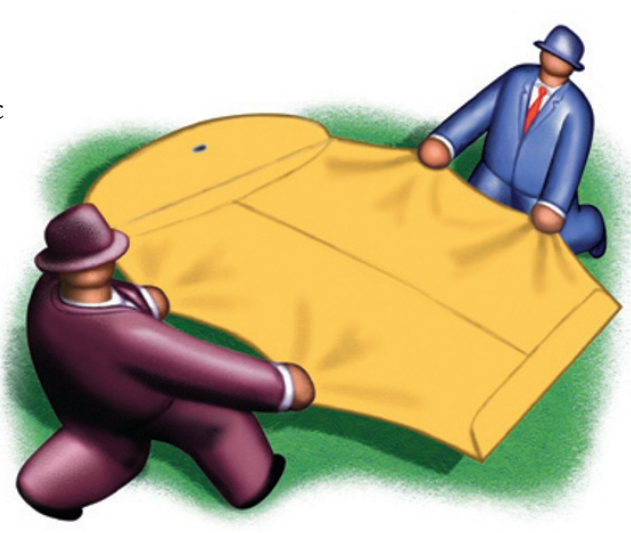

